\title{
Designing coupled coplanar waveguide standards for on-wafer mixed-mode S-parameter measurement
}

\author{
Thi Dao Pham $^{1,2}$, Djamel Allal ${ }^{1}$, François Ziadé $^{1}$ and Eric Bergeault ${ }^{2}$ \\ ${ }^{1}$ Laboratoire national de métrologie et d'essais (LNE), 29 Avenue Roger Hennequin, 78197, Trappes Cedex, France \\ ${ }^{2}$ Télécom ParisTech, Université Paris-Saclay, 46 Rue Barrault, 75013, Paris, France
}

\begin{abstract}
This paper presents the designing of balanced calibration standards for the use of Multimode TRL technique involving the on-wafer Vector Network Analyzer (VNA) measurement of differential devices in the frequency band up to $67 \mathrm{GHz}$. These standards are based on the coupled coplanar waveguide (CCPW) lines in the configuration "Ground-Signal-Ground-Signal-Ground" (GSGSG). The application of the Multimode TRL algorithm on the simulated mixed-mode S-parameters of an attenuator shows the possibility to correct error terms then to de-embed their effects from the device under test (DUT).
\end{abstract}

\section{Introduction}

In high-frequency integrated circuit design, differential circuits have become common in various applications such as low-noise amplifiers (LNA), directional couplers, etc. Prior researches have demonstrated the advantages of a differential device compared to a single-ended circuit in terms of noise suppression and signal stability. Mixed-mode Sparameters were also introduced to describe the differential-mode and common-mode propagations as well as any mode conversions [1]. The symmetric devices are characterized through the mixed-mode Sparameter measurement using a 2- or multi-port VNA.

For the purpose of wafer-level mixed-mode Sparameter measurement, an on-wafer calibration method is essential for the determination and correction of systematic errors in the measurement system such as coaxial cables, radio frequency (RF) probes and imperfections of the VNA. Referencing to the wellknown calibration method Thru-Reflect-Line (TRL) used for asymmetrical measurements [2], a Multimode TRL technique for the calibration of the differential signal measurement is developed to take the multimode propagation into account [3]. Analytical derivation for the Multimode TRL algorithm has been developed using the new generalized cascade matrices that are an extension of the ordinary scattering matrix used in waveguide theory [4].

From the perspective of high-frequency electric metrology, our work focuses on designing and fabricating the calibration kit that meets the error correction accuracy of mixed-mode S-parameters measurements for frequencies up to $67 \mathrm{GHz}$ and allows establishing the S-parameter traceability to the International System of Units (SI).

\section{Multimode TRL standards on CCPW structure}

The Multimode TRL technique is briefly reviewed in the case of differential two-port network to highlight the major steps in the calibration algorithm and to determine the choice of its standards. In fact, the Thru and Line standards are simply the coupled transmission lines with different lengths and meet the requirement that the phase difference between these two standards should be greater than 20 degrees and less than 160 degrees [5]. On the contrary, the Reflect standard has the same high reflection at both VNA ports.

The measurement system is presented by an ideal VNA together with the error boxes A and B at each mixedmode port that include the non-ideality of cables, RF probes, connectors and VNA itself. By presenting these networks in form of generalized cascade matrices, the uncorrected cascade matrix $\mathrm{M}_{\mathrm{x}}$ is the product of three matrices including the corrected cascade matrix $\mathrm{N}_{\mathrm{x}}$ of the DUT and two matrices A and B (Fig. 1).

$$
M_{x}=A \cdot N_{x} \cdot \bar{B}
$$

The overbar of B in the equation (1) is used to describe the cascade matrix B from right to left to take advantage of the symmetry of the whole system.

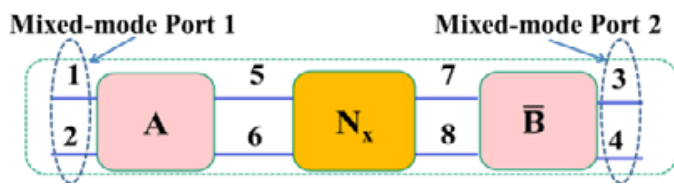

Fig. 1. Model of 4-port measurement system

Corresponding author: thi-dao.bui@1ne.fr

C) The Authors, published by EDP Sciences. This is an open access article distributed under the terms of the Creative Commons Attribution License 4.0 (http://creativecommons.org/licenses/by/4.0/). 
The calibration procedure allows determining the unknown error terms in the matrices $\mathrm{A}$ and $\mathrm{B}$ and then applying the correction to the measurement on the DUT. The Multimode TRL calibration algorithm can be grouped into two main parts.

The first part focuses on the determination of complex propagation constants of both common and differential modes from the measurements of the Thru and Line standards. By introducing two similar matrices (one partially unknown matrix $\mathrm{P}\left(\mathrm{P}=\mathrm{N}_{\mathrm{L}} \cdot \mathrm{N}_{\mathrm{T}}^{-1}\right)$ and one completely known matrix $\mathrm{Q}$ from the uncorrected data $\left.\left(\mathrm{Q}=\mathrm{M}_{\mathrm{L}} \cdot \mathrm{M}_{\mathrm{T}}^{-1}\right)\right)$ that share the same eigenvalues, propagation constants are derived as presented in the equations from (2) to (5).

$$
P=\left[\begin{array}{cccc}
e^{-\gamma_{d m} \cdot \Delta l} & 0 & 0 & 0 \\
0 & e^{-\gamma_{c m} \cdot \Delta l} & 0 & 0 \\
0 & 0 & e^{\gamma_{d m} \cdot \Delta l} & 0 \\
0 & 0 & 0 & e^{\gamma c m \cdot \Delta l}
\end{array}\right]
$$

$\Delta l$ is the difference in lengths between Line and Thru standards $\left(\Delta \mathrm{l}=\mathrm{L}_{\mathrm{L}}-\mathrm{L}_{\mathrm{T}}\right)$.

$$
\begin{gathered}
\operatorname{eig}(P)=\left(e^{-\gamma_{d m} \cdot \Delta l} ; e^{-\gamma_{c m} \cdot \Delta l} ; e^{\gamma_{d m} \cdot \Delta l} ; e^{-\gamma_{c m} \cdot \Delta l}\right) \\
{\left[Y_{0 i}, \lambda_{i}\right]=\operatorname{eig}(Q)=\operatorname{eig}(P), i=1, \ldots, 4}
\end{gathered}
$$

where $\lambda_{\mathrm{i}}$ and $\mathrm{Y}_{0 \mathrm{i}}$ are the eigenvalue and eigenvector of the matrix $Q$, respectively.

$$
\gamma_{i}=\frac{1}{\Delta l} \ln \left(\lambda_{i}\right)
$$

Four values $\gamma_{\mathrm{i}}$ derived from the eigenvalues of the matrix $\mathrm{Q}$ correspond to the two pairs of propagation constants in the forward and backward directions of differential ($\left.\gamma_{\mathrm{dm}}, \gamma_{\mathrm{dm}}\right)$ and common $\left(-\gamma_{\mathrm{cm}}, \gamma_{\mathrm{cm}}\right)$ modes. The assignment of the calculated eigenvalues to the corresponding propagating modes and propagating directions is based on the estimated propagation constants of the simulation or of the analytical calculation. By using the eigenvector $\mathrm{Y}_{0 \mathrm{i}}$ of the matrix $\mathrm{Q}$ derived from the equation (4) and the eigenvalue decomposition of the matrices $\mathrm{P}$ and $\mathrm{Q}$, four unknown constants remain in the determination of the matrices A and B after applying some matrix manipulations [4].

The second part uses the Reflect standard to find out the remaining unknowns in the matrices $\mathrm{A}$ and $\mathrm{B}$ mainly thanks to its identical reflection matrix $\Gamma$ at each measurement port and its reciprocal property $\left(\Gamma=\Gamma^{\mathrm{T}}\right)$. Unlike in the case of traditional TRL calibration, the Reflect standard of the Multimode TRL approach for four-port vector network analyzer calibration is a twoport structure with all non-zero modal S-parameters. This criterion requires that the Reflect standard must be physically asymmetrical to yield mode conversions. In addition, all elements of the modal matrix of Reflect standard must be large enough to improve calibration accuracy. One possible choice for this standard composing of an Open at port 1 and a matched load $\mathrm{Z}_{\mathrm{L}}$ connected to port 2 is shown in the Fig. 2. The theoretical modal reflection matrix of this Reflect standard is presented in the equation (6).

$$
\begin{aligned}
& {\left[\begin{array}{ll}
\Gamma_{d d} & \Gamma_{d c} \\
\Gamma_{c d} & \Gamma_{c c}
\end{array}\right]=\left[\begin{array}{cc}
0.5 & -0.5 \\
-0.5 & 0.5
\end{array}\right]}
\end{aligned}
$$

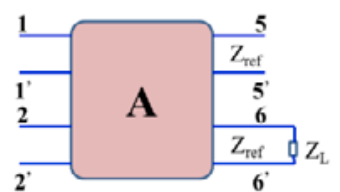

Fig. 2. Open-Load-Open (OLO) Reflect standard

From the analysis of Multimode TRL technique (see [4] for details), the calibration standards are proposed by using the coupled coplanar waveguide in the configuration GSGSG without a lower ground plane (Fig. 3). The advantage of having all of the ground planes $\left(\mathrm{G}_{1}, \mathrm{G}_{2}\right.$ and $\left.\mathrm{G}_{3}\right)$ and strip conductors $\left(\mathrm{S}_{1}\right.$ and $\left.\mathrm{S}_{2}\right)$ on the same side of the substrate is to facilitate the onwafer measurement in the term of deposing the probe's tips. Furthermore, eliminating the need for via holes to a plane on another side of the substrate helps to simplify the fabrication. In addition, a ground plane between the two strip lines minimizes the coupling effect between these conductors.

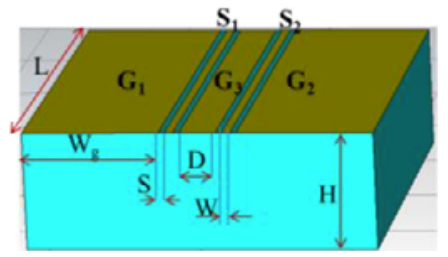

Fig. 3. CCPW in the configuration GSGSG

The dimension (Table 2) and materials (Table 3) of the standards are defined basing on the criteria of impedance matching, wafer surface, RF probe pitch as shown in the Table 1.

Table 1. Criteria for choosing of calibration standards

\begin{tabular}{|c|c|c|c|}
\hline Criterion & $\begin{array}{c}\text { Impedance } \\
\text { matching }\end{array}$ & $\begin{array}{c}\text { Wafer } \\
\text { surface }\end{array}$ & Probe pitch \\
\hline Value & $50 \Omega$ & 2 inches & $100 \mu \mathrm{m}$ \\
\hline
\end{tabular}

Table 2. Dimension of CCPW lines

\begin{tabular}{|c|c|c|c|c|c|}
\hline & S & W & D & $\mathbf{W}_{\mathrm{g}}$ & H \\
\hline $\begin{array}{c}\text { Dimension } \\
(\mu \mathbf{m})\end{array}$ & 20 & 28 & 120 & 450 & 400 \\
\hline
\end{tabular}

Table 3. Materials of CCPW lines

\begin{tabular}{|c|c|c|c|}
\hline & Conductor & Substrate & Resistor \\
\hline Materials & Gold & GaAs & Titan \\
\hline
\end{tabular}


For the traditional TRL calibration method, a single Line standard is usable just over an 8:1 frequency range. Therefore, for broader frequency range from $1 \mathrm{GHz}$ up to $67 \mathrm{GHz}$, at least three Line standards with different lengths need to be used together with the Thru standard. The equation (7) is used to determine the length of these three lines in each frequency range $\left[\mathrm{f}_{1}: \mathrm{f}_{2}\right]$ in condition that the effective dielectric constant of the differential and common modes $\varepsilon_{\text {eff(dm,cm) }}$ at the central frequency $\mathrm{f}_{\mathrm{c}}$ $\left(\frac{f_{1}+f_{2}}{2}\right)$ is known.

$$
\Delta L_{(d m, c m)}=L_{\text {Line }}-L_{\text {Thru }}=\frac{c}{2 \sqrt{\varepsilon_{e f f(d m, c m)}}\left(f_{1}+f_{2}\right)}
$$

where $\mathrm{c}$ is the speed of light in vacuum.

For the estimation of lines' length, we use only the static value of effective permittivity that is calculated as the ratio between the total capacitance per unit length $\mathrm{C}_{\mathrm{t}(\mathrm{dm}, \mathrm{cm})}$ and the capacitance in the absence of the dielectric layer $\mathrm{C}_{\mathrm{a}(\mathrm{dm}, \mathrm{cm})}$ for each mode.

$$
\varepsilon_{e f f(d m, c m)}=C_{t(d m, c m)} / C_{a(d m, c m)}
$$

The estimated effective dielectric constants of differential and common modes are 6.948 and 6.949, respectively. In this case, $\mathrm{C}_{\mathrm{t}(\mathrm{dm}, \mathrm{cm})}$ and $\mathrm{C}_{\mathrm{a}(\mathrm{dm}, \mathrm{cm})}$ are simulated by the COMSOL Multiphysics software [6].

By fixing the length of Thru standard as $500 \mu \mathrm{m}$, the length of three Line standards $\mathrm{L}_{1}, \mathrm{~L}_{2}$ and $\mathrm{L}_{3}$ are summarized in the table below:

Table 4. Lines' length corresponding to each frequency range

\begin{tabular}{|c|c|c|c|}
\hline & $\mathbf{L}_{\mathbf{1}}$ & $\mathbf{L}_{\mathbf{2}}$ & $\mathbf{L}_{\mathbf{3}}$ \\
\hline $\begin{array}{c}\text { Length of lines } \\
(\boldsymbol{\mu} \mathbf{m})\end{array}$ & 6823 & 3661 & 1202 \\
\hline $\begin{array}{c}\text { Frequency range } \\
{\left[\mathbf{f}_{\mathbf{1}}: \mathbf{f}_{\mathbf{2}}\right] \mathbf{( G H z )}}\end{array}$ & $1: 8$ & $2: 16$ & $9: 67$ \\
\hline
\end{tabular}

Relating to the Reflect standard, an Ohmic resistance realized in titan is used to create the load $R_{L}$ of $50 \Omega$.

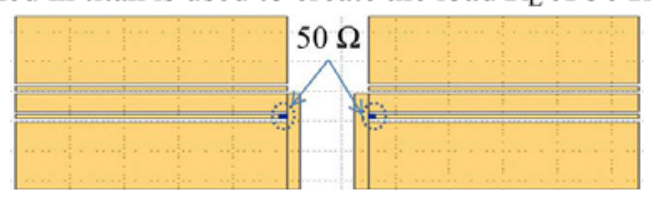

Fig. 4. OLO Reflect standard using CCPW

Since the sheet resistance per square $R_{S}$ is also $50 \Omega$, the load in the square form spotted on the strip line has the same value of width $\left(\mathrm{W}_{\mathrm{L}}\right)$ and length $\left(\mathrm{L}_{\mathrm{L}}\right)$ (Fig. 4).

$$
L_{L}=W_{L} \cdot \frac{R_{L}}{R_{S}}=28 \frac{50}{50}=28(\mu \mathrm{m})
$$

\section{Validation of the Multimode TRL algorithm}

To demonstrate the feasibility of standards presented above for the error correction of Multimode TRL calibration method, different DUTs such as adapted CCPW lines, mismatched CCPW lines and attenuators are considered. In this paper, a doubled $10 \mathrm{~dB} \mathrm{~T}$ attenuator is used to verify the Multimode TRL algorithm (Fig. 5).
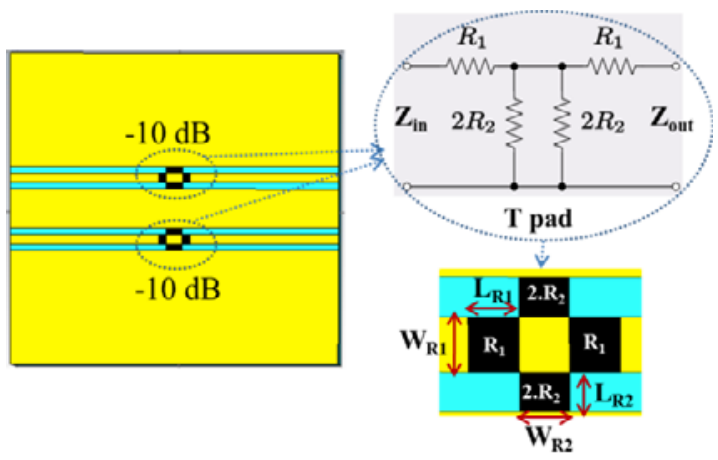

Fig. 5. Doubled $10 \mathrm{~dB}$ T-attenuator

With the same reference impedance $Z_{\text {ref }}$ for the input and ouput sides $\left(Z_{\text {ref }}=Z_{\text {in }}=Z_{\text {out }}\right)$, the value of the resistances $R_{1}$ and $R_{2}$ is calculated by the equation (10).

$$
R_{1}=Z_{r e f} \cdot \frac{1-N}{N+1} ; R_{2}=2 . Z_{r e f} \cdot \frac{N}{1-N^{2}}
$$

For a $10 \mathrm{~dB} \mathrm{~T}$-attenuator, the attenuation coefficient $\mathrm{N}$ is $0.316\left(\mathrm{~N}=10^{-(10 / 20)}\right)$. Assuming that $Z_{\text {ref }}$ is equal to $50 \Omega$, the value of the resistances $R_{1}$ and $R_{2}$ are $25.97 \Omega$ and $35.13 \Omega$. To form these resistance values, their dimension is as follows:

Table 5. Dimension of resistances $R_{1}$ and $R_{2}$

\begin{tabular}{|l|c|c|c|c|}
\hline & $\mathbf{W}_{\mathbf{R} 1}$ & $\mathbf{L}_{\mathbf{R} 1}$ & $\mathbf{W}_{\mathbf{R} 2}$ & $\mathbf{L}_{\mathbf{R} 2}$ \\
\hline Length $(\boldsymbol{\mu m})$ & 28 & 15 & 14 & 20 \\
\hline
\end{tabular}

To obtain the DUT's mixed-mode S-parameters by simulation with CST Microwave Studio, the waveguide port with Multipin is used. This DUT is also simulated by putting it between two error boxes $\mathrm{A}$ and $\mathrm{B}$ at each mixed-mode port 1 and 2. For simplicity, the networks A and $\mathrm{B}$ are illustrated by the CCPW lines with the length of $5000 \mu \mathrm{m}$ for each one (Fig. 6).

\begin{tabular}{|c|c|c|}
\hline $\mathrm{A}$ & DUT & \\
\hline \hline $\mathrm{L}_{\mathrm{A}}$ & & $\mathrm{B}$ \\
\hline
\end{tabular}

Fig. 6. Simulation of DUT and error networks A and B

The figure 7 and figure 8 show the comparison of the mixed-mode S-parameters in two cases: one is of the DUT simulation (denoted by red triangles); another is of the simulation for the whole system (DUT and error 
networks A and B) then applying the Multimode TRL algorithm (denoted by blue circles).

Here, $\mathrm{S}^{\mathrm{dd}}{ }_{12}$ and $\mathrm{S}^{\mathrm{cc}}{ }_{12}$ describe the differential and common transmissions from mixed-mode port 2 to mixed-mode port 1. Similarly, $\mathrm{S}^{\mathrm{dd}}{ }_{11}$ and $\mathrm{S}_{11}^{\mathrm{cc}}$ describe differential and common mode reflections of the mixedmode port 1 .

As presented in Fig. 7, both cases provide the similar results of transmission factors. It can be interpreted that applying the Multimode TRL calibration helps remove errors introduced in the error boxes $\mathrm{A}$ and $\mathrm{B}$.

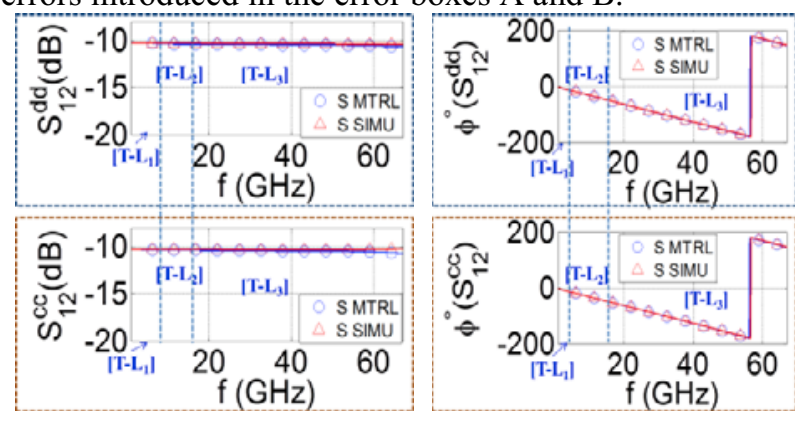

Fig. 7. Transmission coefficients: input port 1, output port 2
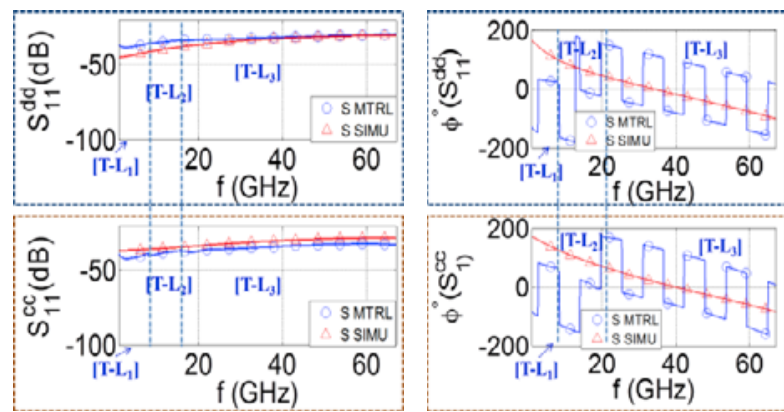

Fig. 8. Reflection coefficients of mixed-mode port 1

For the reflection coefficients, we may not be able immediately to draw the conclusion for the possibility of applying this calibration for the correction of error terms by using these simulated data. This problem can be explained that the simulated S-parameters in CST simulation depend on the definition and the nature of waveguide port, that doesn't take the frequencydependent effects into account.

In regards to mode-conversion S-parameters, thanks to the symmetry of the DUT, the phenomenon of mode conversion is ignored (less than $-100 \mathrm{~dB}$ in magnitude) in the frequency band of interest.

\section{Conclusions}

This paper presents the designing of Multimode TRL based on the coupled coplanar waveguide in the configuration GSGSG. Three CCPW transmission lines with different lengths are used together with the Thru and Reflect standards to cover the frequency band from $1 \mathrm{GHz}$ to $67 \mathrm{GHz}$. The application of Multimode TRL algorithm on the simulated S-parameters of the doubled $10 \mathrm{~dB}$ T-attenuator as symmetric DUT demonstrates the feasibility of Multimode TRL calibration. This calibration method allows figuring out the propagation constants of both common and differential modes and then reducing error terms to obtain the corrected data. The results show the agreement between the simulated transmission factors of DUT and the transmission factors after applying the Multimode TRL on the uncorrected Sparameters. As the calibration kit is under fabrication, the next step is to work on the measurement data by using the on-wafer VNA measurement system to measure different types of DUTs in order to verify the possibility of Multimode TRL calibration to correct the whole mixed-mode S-parameters.

\section{References}

1. D.E. Bockelman, W.R. Eisenstadt, IEEE Trans. on MW Theory and Techniques 43(7), 1530-1539 (1995).

2. G.F. Engen, C.A. Hoer, IEEE Trans. on MW Theory and Techniques 27(12), 987-993 (1979).

3. C. Seguinot, P. Kennis et al., IEEE Trans. on MW Theory and Techniques 46(5), 536-542(1998).

4. M. Wojnowski, V. Issakov, G. Sommer, \& R. Weigel, IEEE Trans. on MW Theory and Techniques 60(7), 2220-2247, (2012).

5. R.B. Marks, IEEE Trans. on MW Theory and Techniques 39(7), 1205-1215 (1991).

6. A.B. Comsol, "AC/DC Module-User's Guide." COMSOL 3, 151 (2011). 